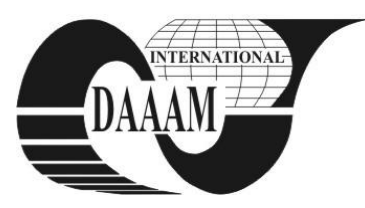

Annals of DAAAM for 2011 \& Proceedings of the 22nd International DAAAM Symposium, Volume 22, No. 1, ISSN 1726-9679 ISBN 978-3-901509-83-4, Editor B. Katalinic, Published by DAAAM International, Vienna, Austria, EU, 2011 Make Harmony between Technology and Nature, and Your Mind will Fly Free as a Bird Annals \& Proceedings of DAAAM International 2011

\title{
METAMATERIAL BASED MICROWAVE BAND-PASS FILTER
}

\author{
DE SABATA, A[Ido]; MATEKOVITS, L[adislau]; ROHDE, U[Irich] L. \& SILAGHI, M[arius] A[lexandru]
}

\begin{abstract}
A passive, low-cost device built with a textured surface, consisting of a periodic lattice of metal patches, embedded in an inhomogeneous parallel-plate waveguide is proposed. It acts as a band-pass filter in the microwave frequency range and it can be also used in sensor applications. The frequency related properties of the structure is presented by means of the dispersion diagram calculated by full-wave electromagnetic simulation. A parametric study in function of the dielectric constant of one of the materials entering the construction of the device is performed.

Key words: metamaterials, high impedance surface, electromagnetic band gap, band-pass filtering
\end{abstract}

\section{INTRODUCTION}

Metamaterials are man-made materials that have properties not found in nature (Lapine \&Tretyakov, 2007). The interest in microwave related applications of metamaterials have been fostered by interesting potential realizations of High Impedance Surfaces (HISs) based on microstrip technology for low profile antennas (Sievenpiper et al., 1999). HISs are usually realized by impressing a periodic pattern on one face of a microstrip board. Such planar structures present an Electromagnetic Band-Gap (EBG) in the frequency domain, where surface waves cannot propagate and the surface impedance is very high, so that the structure behaves like an artificial magnetic conductor.

The presence of an EBG suggests applications of HISs to filters. In the past, several periodic structures have been proposed (Gao et al., 2006, Karmalar \& Mollah, 2003, Menzel et al., 2003).

Following the development of wireless technology in the last years, the necessity of simple, low-cost compact filters having several pass- and stop-bands has occurred. HISs having several EBGs have yet been proposed to accomplish such requirements. For example, in (Hao et al., 2009) a periodical, slotted ground structure is devised, while in (Zhang el al., 2008) multi-band filters are obtained by cascading unit cells taken from different HISs.

In this paper, we propose a HIS, embedded in an inhomogeneous parallel-plate waveguide (PPW), that presents two EBGs, one from DC to a cutoff frequency, denoted $f_{0}$ and a second one between two cutoff frequencies denoted $f_{1}$ and $f_{2}$ respectively. Consequently, the structure conducts surface waves in the $\left(f_{0}, f_{1}\right)$ range that can even be quite narrow if properly designed. Therefore, the device may be used for bandpass filtering and for sensor applications.

The properties of the proposed electromagnetic object are derived by full-wave electromagnetic simulation, by means of a commercial field solver (CST Microwave Studio). During the numerical simulations the symmetry of the proposed structure have been considered and the dispersion diagrams (DDs) on the whole GXMG border of the first irreducible Brillouin zone (Brillouin 1953) have been calculated. It allows revealing all relevant frequency related properties.

\section{PRESENTATION OF THE DEVICE}

The structure of the device we are proposing is reported in Fig. 1 (a). It consists of two parallel metal plates that contain in between two different media of dielectric constants $\varepsilon_{r 1}$ and $\varepsilon_{r 2}$ respectively. At the border of the two media, a textured surface consisting of metal patches arranged in a periodic $2 \mathrm{D}$ lattice is impressed. Circular shaped patches have been chosen for each unit cell of the lattice. Metal pins connect the upper metal plate to the patches and vias connect the lower metal plate to the same patches, one of each for every unit cell. We have chosen pins instead of vias for the connection of the upper plate as $\varepsilon_{r 2}$ may be equal to unity, so that air fills the corresponding space. Therefore, the mechanical structure is more robust in this case. A unit cell of the textured surface is represented in Fig. 1 (b) and the HIS obtained by the repetition of the unit cell is reported in Fig. 1 (c). Fig. 1 (d) shows the CAD model used for the unit cell of the device.

The following parameters have been used for the numerical investigations: dimension of the square shaped unit cell $d=2.5 \mathrm{~mm}$, patch diameter $2 r=2.1 \mathrm{~mm}, t_{1}=1.6 \mathrm{~mm}, t_{2}=0.3 t_{1}$, radius of vias $0.1 \mathrm{~mm}$, radius of pins $0.15 \mathrm{~mm}$. The dielectric constant of the lower layer has been kept constant $\varepsilon_{r 1}=3.5$, while that of the upper layer, $\varepsilon_{r 2}$, has been varied between 1 and 12 during simulations, clearly targeting sensor like applications.

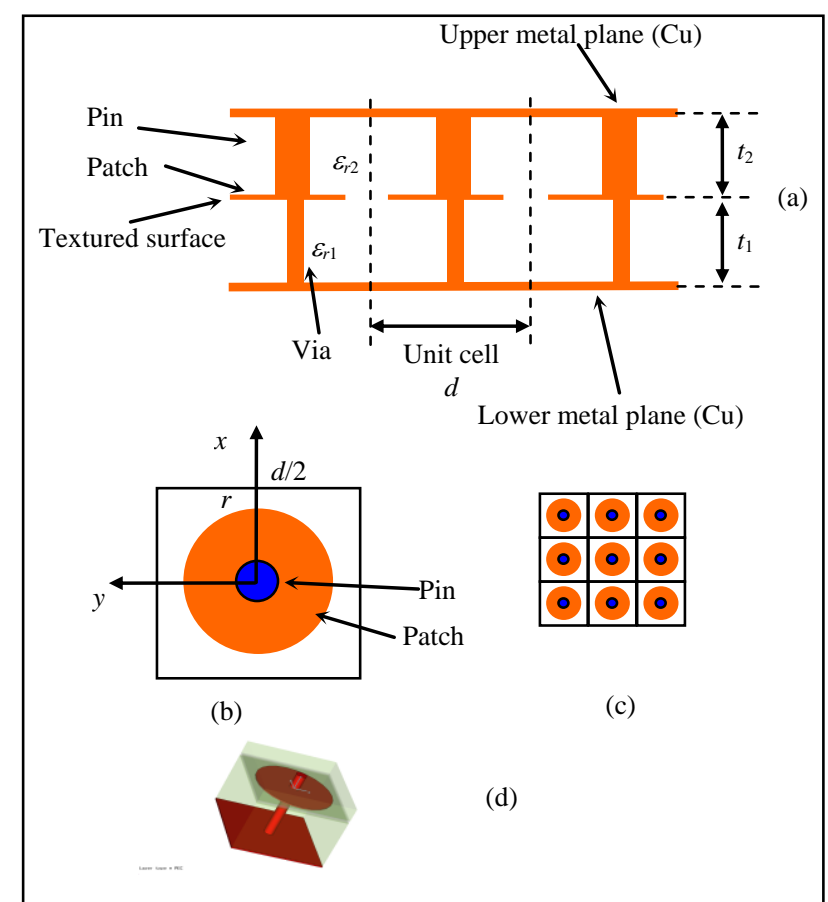

Fig. 1. (a) Proposed structure; (b) unit cell of the textured surface; (c) textured surface; (d) CAD model with upper metal plane removed for visualization

\section{RESULTS}

The DD (frequency of modes versus wavenumber) of the structure for the case $\varepsilon_{r 2}=12$ is reported in Fig. 2. The shape of the border of the first irreducible Brillouin zone in the wavenumbers space is drawn in the inset of the figure. Light- 
lines (LLs) are also represented, by assuming an effective dielectric constant of the inhomogeneous medium defined by $\varepsilon_{r 1}$ and $\varepsilon_{r 2}$ (Rogers, 2005). LLs are used for indicating separation between fast and slow surface waves.

The first EBG spreads from DC up to $f_{0}$, which is the minimum frequency of the first mode. The second EBG is defined by $f_{1}$, the maximum frequency of the first mode, and $f_{2}$ the minimum frequency of the second mode. The fact that low frequency waves are cut up to DC is motivated by the DC connection between the two metal plates.

The frequency positions and widths of the EBGs can be adjusted according to requirements of various applications by varying the geometrical and material parameters of the device. A study on the influence of the dielectric constant of the upper layer on the relevant frequencies $f_{0}, f_{1}$ and $f_{2}$, have been performed, and some results are reported in Fig. 3. This choice is motivated by availability of a large range of low loss materials for high frequency applications (She et al., 2005)

The frequency span is clearly sensitive to the material of the upper layer. The width of the pass-band (between $f_{0}$ and $f_{1}$ ) diminishes with the increase of the dielectric constant, making the filter more selective.

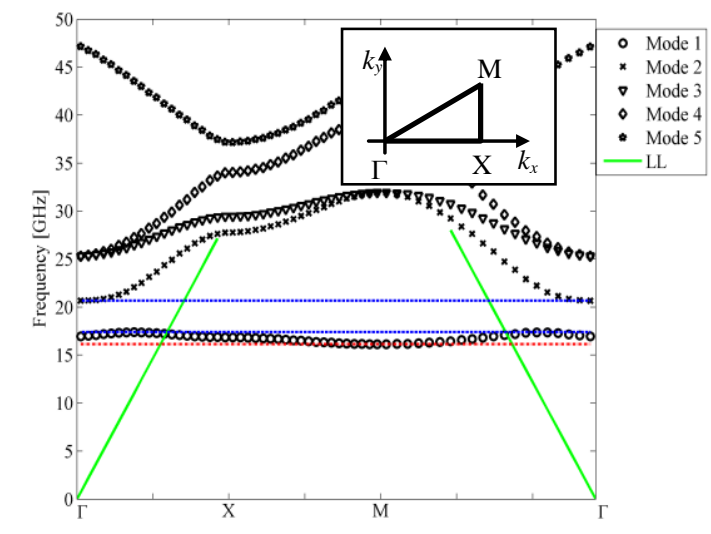

Fig. 2. DD of the proposed structure, featuring the first five modes. Inset: border of the first irreducible Brillouin zone

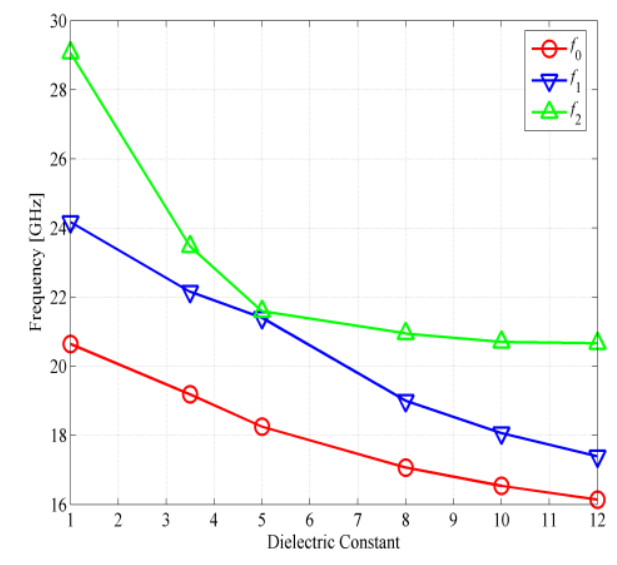

Fig. 3. Frequency limits of the EBGs versus dielectric constant of the upper material layer

\section{CONCLUSION}

A passive, low-cost electromagnetic structure that can be used a pass-band filter in the $\mathrm{GHz}$ range has been proposed.
The construction of the device relies on strip-line technology; it is an inhomogeneous PPW containing a HIS that features two EBGs for propagation of surface waves in between the first three modes. We have used the DDs for presenting the frequency related properties of the structure and we have performed a parametric study in function of the dielectric constant of one of the materials entering the construction of the device in order to demonstrate the flexibility of the design.

Several other parametric studies, versus the geometric parameters are scheduled to be performed in the future in order to provide further degrees of freedom in design.

\section{ACKNOWLEDGMENT}

The research of Ladislau Matekovits has been supported by a Marie Curie International Outgoing Fellowship within the 7th European Community Framework Programme.

\section{REFERENCES}

Ahn D.; Park J.-S.; Kim C.-S.; Kim J.; Qian Y. \& Itoh T (2001). A design of the low-pass filter using the novel microstrip defected ground structure, IEEE Trans. Microwave Theory Tech., vol. 49, no. 1, January 2001, pp. 86-93, ISSN 0018-9480

Brillouin L (1953). Wave Propagation in Periodic Structures, Dover, ISBN n/a, New York

Gao C.; Chen Z. N.; Wang Y. Y.; Yang N. \& Qing X. M. (2006). Study and suppression of ripples in passbands of series/parallel loaded EBG filters, IEEE Trans. Microwave Theory Tech., vol. 54, no. 4, April 2006, pp. 1519-1525, ISSN 0018-9480

Hao Z.-C.; Hong J.-S.; Parry J. P. \& D. P. Hand (2009). Ultrawideband bandpass filter with multiple notch bands using nonuniform periodical slotted ground structure, IEEE Trans. Microwave Theory Tech., vol. 57, no. 12, December 2009, pp. 3080-3088, ISSN 0018-9480

Karmakar N. C. \& Mollah M. N. (2003). Investigation into nonuniform photonic band-gap microstripline low-pass filters, IEEE Trans. Microwave Theory Tech., vol. 51, no. 2, February 2003, pp. 564-572, ISSN 0018-9480

Lapine M. \& Tretyakov S. (2007), Contemporary notes on metamaterials (2007), IET Microw. Antennas Propag., 1, (1), 2007, pp.3-11, ISSN 1751-8725

Menzel W.; Zhu L.; Wu K. \& Bögelsach F. (2003), On the design of novel compact broad-band planar filter, IEEE Trans. Microwave Theory Tech., vol. 51, no. 2, February 2003, pp. 364-369, ISSN 0018-9480

Rogers S. D. (2005) Electromagnetic-bandgap layers for broadband suppression of TEM modes in power planes, IEEE Trans. Microw. Theory Tech., vol. 53, no. 8, Aug. 2005, pp. 2495-2505, ISSN 0018-9480

She W. H.; Wing Z. N.; Halloran J. W.; Chappell W. J. (2005), Variable dielectric constants by structured porosity for passive ceramic components, Digest of 2005 IEEE MTT-S International Microwave Symposium, pp. 865-869, 12-17 June 2005

Sievenpiper D.; Zhang L.; Boas F. J.; Alexópoulos N. G. \& Yablonovitch E. (1999). High-impedance electromagnetic surfaces with a forbidden frequency band, IEEE Trans. Microwave Theory Tech, vol. 47, no. 11, Nov. 1999, pp. 2059-2074, ISSN 0018-9480

***Zhang L.-J.; Liang C.-H.; Liang L. \& Chen L (2008). A novel design approach for dual-band electromagnetic bandgap structure, Progress in Electromagnetics Research, vol. 4, pp. 81-91, 2008 ISSN 1070-4698

***CST Microwave Studio (2009) 